\title{
On the Definition of Resource Sharing Levels to Understand and Control the Impact of Contention in Multicore Processors
}

\author{
Hamid Tabani and Leonidas Kosmidis Barcelona Supercomputing Center
}

Enrico Mezzetti and Jaume Abella Barcelona Supercomputing Center and Maspatechnologies S.L.

Francisco J. Cazorla Barcelona Supercomputing Center: Centro Nacional de Supercomputacion

Citation: Tabani, H., Kosmidis, L., Mezzetti, E., Abella, J. et al., "On the Definition of Resource Sharing Levels to Understand and Control the Impact of Contention in Multicore Processors," SAE Technical Paper 2021-01-5055, 2021, doi:10.4271/2021-01-5055.

\section{Abstract}

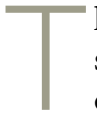

he trend toward the adoption of a multiprocessor system on a chip (MPSoC) in critical real-time domains, like avionics or automotive, responds to the demand for increased computing performance to support advanced software functionalities. The other side of the coin is that MPSoCs challenge software timing analysis. This is so as co-running applications affect each other's timing behavior on account of the interference incurred when accessing shared hardware resources, with the latter steadily increasing in number and complexity in every new generation of MPSoCs. For a solid and cost-contained software-timing validation approach, we contend that a taxonomy has to be developed to capture the different levels at which processors' resources can

\section{Keywords}

Multicore timing analysis, Multicore contention, Software timing, Freedom from interference, Interference channel be shared. Those levels are to be related to the conventional run-time software abstractions (e.g., task, thread, runnable) and the particular abstraction used to carry out contention analysis. From the standpoint of contention analysis, only the resources in those levels shared by the different run-time software entities need to be mastered and addressed by timing analysis, whereas the remaining resources can be safely disregarded.

We tailor this approach to two of NVIDIA's embedded platforms, TX2 and AGX Xavier, of particular relevance for the automotive domain. For the identified shared resources, we also characterize the contention that tasks can suffer and discuss the limitations and early approaches for modeling timing interference in shared hardware resources.

\section{Introduction}

T

he need for increased computing performance in all embedded critical-system markets like automotive and avionics is relentless $[1,2]$. This is fueled by the adoption of advanced software-controlled functionalities in new products where software is required to process massive and diverse data (e.g., coming from sensors like cameras, radars, and lidars) and to implement complex control functions, building on machine-learning algorithms. For instance, in the automotive domain, car manufacturers and technology providers have started analyzing the viability of mass production of Autonomous Driving (AD) cars.

MultiProcessor Systems on Chips (MPSoCs), comprising multicore CPUs and accelerators like Graphics Processing Units (GPUs), are at the forefront of the solutions used in these high-performance real-time systems to deliver high computing performance at a reasonable cost $[\underline{1}, \underline{3}, \underline{4}, \underline{5}]$. While MPSoCs can incorporate specific features for their target market, - they largely build on designs originally devised for the mainstream market as a means to reduce nonrecurring (engineering) costs. As a drawback, however, mainstream solutions are typically not designed with predictability in mind, challenging the software timing verification. The source of the problem lies in the contention in the access to hardware-shared resources, e.g., caches, that makes the execution time of a task dependent on the tasks simultaneously running in the MPSoC. To make things worse, current (and future) MPSoCs exhibit complex

${ }^{1}$ Few chip vendors (e.g., Intel and NVIDIA) already have automotive-specific divisions to market variants of their mainstream architectures. 
architectures with many shared resources, challenging the state of play of static and measurement-based timing analysis techniques. In the face of these challenges, solutions are heavily sought for conducting early-stage characterization of contention effects, even for those advanced functionalities (and configurations) that cannot be analyzed with conventional analytical models [6]. We contend that an early characterization of contention effects can be obtained by focusing on the different types and levels of resource sharing in a given platform.

For software timing verification, safety standards and support documents provide guidelines for complex multicore platforms. For instance, Certification Authorities Software Team (CAST)-32A [7] recommends identifying each interference channel in the target platform, such as caches or buses, and bounding the potential impact they may have on tasks timing. The International Organization for Standardization (ISO) 26262 standard Part 6 [] $]$ mandates freedom from interference (FFI) to avoid timing faults across software elements (e.g., tasks), and, in particular, those caused by low-Automotive Safety Integrity Level (ASIL) tasks on high-ASIL ones. However, how to adhere to these guidelines for a specific SoC remains an open challenge. In this work, we contribute to reducing the gap between guidelines and practice for software timing on modern GPU-based SoCs as follows:

Definition of a Resource Sharing Level (RSL) Taxonomy. We present a taxonomy of shared hardware resources in a heterogeneous SoC, with the goal of identifying the Resource Sharing Levels (RSLs) in which different software components can compete in the access. Our taxonomy covers multicore central processing units (CPUs), GPUs, and specific accelerators: (i) In CPUs, resource sharing ranges from intra-CPU and intra-cluster to intra-SoC; (ii) In GPUs RSLs vary from intrastreaming multiprocessor block (SMB) to intra-SoC; (iii) In the SoCs we have analyzed, accelerators do not share internal resources and only share (with the CPU and GPU) the main memory and, sometimes, the memory access channels. We also show how our taxonomy can be tailored for the resource sharing observed in one of the latest NVIDIA SoCs for automotive.

Identification of Relevant RSL. We show that relating the proposed RSL taxonomy with the software abstractions is a necessary step to contain analysis costs. This is so as modern MPSoCs have many shared resources, some of them with intricate dependencies, and simply performing contention analysis in all of them can incur unaffordable costs. Instead, contention analysis should be performed in those RSLs where contention can affect the Unit of Analysis (UoA). For NVIDIA GPUs, for instance, this requires relating the UoA with the software abstractions-which, for NVIDIA devices, includes tasks, streams, kernels, and threads-and the concurrency allowed by the Operating System (OS) or run-time support (e.g., Compute Unified Device Architecture (CUDA) run-time system). Determining those RSLs where different UoAs can contend is as important as containing software timing verification costs: conflicts occurring between

${ }^{2}$ UoA refers to the software component at which WCET is performed (e.g., task or runnables in AUTOSAR for road vehicles and applications (partitions) or processes in ARINC 653 for commercial avionics. different software components of a UoA (e.g., threads) pertain to the Worst-Case Execution Time (WCET) analysis of that UoA and not to the so-called intertask (inter-UoA) contention analysis.

Quantifying and Modeling Contention. We tailor our RSL taxonomy to one of the latest NVIDIA heterogeneous SoCs, the Xavier, and we identify the particular resources and RSL in both the CPU and GPU parts in which contention can occur. In those resources where contention can arise, we characterize the amount of interference that can possibly be incurred at run time. While this cannot be and is not intended to be an exhaustive interference analysis, it provides an early assessment of how the considered platform permits or prevents contention effects. This is, to our knowledge, the first timing interference analysis of NVIDIA Xavier SoC, which is integrated into the automotive Jetson AGX Xavier, NVIDIA DRIVE Xavier, and Drive Pegasus boards. In doing our analysis, we also compare the AGX Xavier with the predecessor Parker SoC (as implemented in the Jetson TX2 board). Our results show that (i) Contention is high for L2 and $\mathrm{L} 3$ cache-intensive tasks at the intra-CPU-cluster level in the Parker and Xavier SoCs, respectively; (ii) Similar trends are observed in both SoCs for tasks making intensive use of the L2 in the GPU, suggesting that tasks of these characteristics should be prevented from running simultaneously in the same cluster/GPU; (iii) Contention is moderate, and while it must be controlled, it does not compromise the benefits of parallel execution. Hence, increasing the number of parallel tasks in SoCs offers a good path to increase the overall performance despite contention; (iv) Interference grows linearity with the number of contenders in the platform, which facilitates contention modeling.

The rest of the paper is organized as follows: Section 2 presents our RSL taxonomy and tailors it to the TX2 and AGX Xavier. Section 3 discusses the RSL on the CPU and the GPU of the TX2 and AGX Xavier shared between UoA. For the shared resources in those RSLs, we discuss the challenges to master contention. Section 4 quantifies the impact that contention has on the identified relevant RSL. Section 5 summarizes the main related works. Finally, Section 6 presents our main conclusions.

\section{RSL Taxonomy}

The advent of multicores and GPUs ${ }^{\underline{3}}$ in time-sensitive domains has increased the impact that contention in accessing shared hardware resources may have on the software timing behavior. Contention in the memory hierarchy has been reported to increase application execution time by a factor of $2 \times$ and $5 \times$ in real processors for space [ 9 ] and automotive [10], with similar results reported in other real-time domains. In fact, mastering contention is one of the main challenges for multiprocessor and GPU SoC adoption. In this work, we focus on contention arising in the main path from the cores and GPU to the main memory, while we do not cover I/O devices.

${ }^{3}$ Table 1 lists the main terms used throughout this paper. 
TABLE 1 Terms used in this work.

\begin{tabular}{|c|c|c|c|}
\hline Term & Definition & Term & Definition \\
\hline GPU & $\begin{array}{l}\text { Graphics Processing } \\
\text { Unit }\end{array}$ & UoA & Unit of Analysis \\
\hline RSL & Resource Sharing Level & SoC & System on Chip \\
\hline$A D$ & Autonomous Driving & ASIL & $\begin{array}{l}\text { Automotive Safety } \\
\text { Integrity Level }\end{array}$ \\
\hline SM & $\begin{array}{l}\text { Streaming } \\
\text { Multiprocessor }\end{array}$ & MPS & Multi-Process Service \\
\hline SMB & SM Block & QM & Quality Management \\
\hline OS & Operating System & DLA & $\begin{array}{l}\text { Deep Learning } \\
\text { Accelerator }\end{array}$ \\
\hline WCET & $\begin{array}{l}\text { Worst-Case Execution } \\
\text { Time }\end{array}$ & $\mathrm{FFI}$ & $\begin{array}{l}\text { Freedom From } \\
\text { Interference }\end{array}$ \\
\hline IL1 & L1 Instruction Cache (I\$) & DL1 & L1 Data Cache (D\$) \\
\hline SU & Specialized Unit & CSF & CPU Switch Fabric \\
\hline ROP & Raster Operator & AT & Analysis Task/Kernel \\
\hline $\mathrm{CT}$ & Contender Task/Kernel & GPC & $\begin{array}{l}\text { Graphics Processing } \\
\text { Cluster }\end{array}$ \\
\hline
\end{tabular}

\subsection{Generic RSL}

Many SoCs contain a multicore processor (CPU) component, a GPU component, and specific accelerators such as deep learning accelerator (DLA) and video encoders/decoders that are included to efficiently perform some specialized operations. For a clearer understanding of how sharing those resources can affect timing, we define different RSLs. While in this work we focus on the NVIDIA family of SoCs, the same methodology can be applied to categorize RSLs for any target platform. Interestingly, RSLs are not the same for GPU and CPU, with both sharing some levels, as shown in Figure 1. For the sake of clarity, and to avoid confusion with software terms, we use hardware thread to refer to a flow of instructions in the processor. At the software level, this can be an Automotive Open System Architecture (AUTOSAR) executable, an Aeronautical Radio Inc. (ARINC) 653 (software) partition, a GPU kernel, etc.

CPU. For the CPU, also referred to as CPU-complex (CCPLEX), we define the following RSLs:

1. Intra-core. Some CPUs allow several hardware threads to be active at the same time and run concurrently, sharing resources like branch predictors, instruction and data caches, issue logic, and on-core functional units.

2. Intra-cluster. For scalability reasons, cores are organized into clusters. Cores in a cluster share oncluster communication channels, usually buses, as the

FIGURE 1 Proposed taxonomy of RSL.

\begin{tabular}{|c|c|c|c|}
\hline CCPLEX & GPU & Accelerator $_{i}$ & Accelerator $_{j}$ \\
\hline \begin{tabular}{l|l} 
Intra-Core \\
\end{tabular} & Intra-SMB & Intra- Acc $_{i}$ & Intra-Acc ${ }_{j}$ \\
\hline Intra-Cluster & Intra-SM & & \\
\hline Intra-CCPLEX & Intra-GPC & & \\
\hline & Intra-GPU & & \\
\hline
\end{tabular}

(c) Hamid Tabani, Leonidas Kosmidis, Enrico Mezzetti. Jaume Abella, Francisco J. Cazorla. number of cores per cluster is limited. Cores in a cluster also share one or several cache memory levels.

3. Intra-CCPLEX. At the highest level, all clusters usually share a coherence fabric and a cache.

The interactions occurring between hardware threads at the innermost level, i.e., in intra-core resources, are much more frequent than those occurring in the intra-CCPLEX level. Yet in terms of impact (slowdown) on applications' execution time, no rule can be established since conflicts in the outermost RSL, while less frequent, might carry extremely long delays for accessing intra-SoC or off-SoC resources such as memory. This calls for the characterization of the contention on an RSL-basis level.

GPU. With a focus on NVIDIA GPUs, we define the following RSLs.

1. Intra-SMB. Each $\mathrm{SM}$, for scalability reasons, can be divided into several processing blocks (SMB). Within an SMB, hardware threads can share instruction (L0) caches or buffers, dispatch units, and general-purpose (execution and load-store) and specialized units (SUs, e.g., transcendental function units and tensor cores). Hence, interactions happen frequently, even at a single cycle level.

2. Intra-SM. The degree of interaction between the hardware at SM level reduces with respect to (w.r.t.) SMB, with threads usually sharing (L1) instruction and data caches and shared memory, in addition to the on-SM data path to access the next cache levels. SMs are analogous to CPU cores in the above CPU RSL taxonomy.

3. Intra-Graphics Processing Clusters (GPC). Equivalently to CPU clusters, SMs are organized into GPC, which also include specific hardware blocks for graphics processing, such as Raster Operators (ROPs).

4. Intra-GPU. Across GPCs, i.e., at the GPU level, hardware threads might share the last level of cache (L2) and the logic to access system memory.

NVDLA and Other Accelerators. There is an increasing number of accelerators (e.g., the DLA in the Xavier SoC) that improve the performance and efficiency of frequently executed specialized operations. In the platforms we examined, accelerators do not allow concurrent execution of multiple hardware threads. However, nothing prevents future SoCs from sharing them, if chip vendors detect that they are not fully utilized by a single hardware thread and hence, sharing can improve resource utilization. In this work, we focus on NVIDIA's deep learning accelerator (NVDLA) block, which provides a simple, flexible, and robust inference acceleration solution. Xavier SoC has two NVDLAs that can be configured to run deep learning workloads. Each NVDLA is set up and operates independently from other NVDLAs, and there is no RSL between them. For this reason and for the sake of clarity, we focus on the use of only one NVDLA unit.

SoC. At the outermost level on the SoC, or Intra-SoC level, resources common to the CCPLEX, GPU, and different accelerators are shared, including the communication means to access memory. 


\section{RSLs and Software Abstractions}

The complexity of the modern SoCs considered in critical real-time domains is overwhelming. One of the main challenges addressed by software timing analysis tool providers, TIER-1 companies, and OEMs is the identification of interference channels, i.e., those shared resources where contention can arise. We address this challenge by identifying the RSLs in which different software UoAs can come into conflict. Hence, instead of "blindly" analyzing all potential interference channels in all RSLs, we restrict the focus on those RSLs shared by different UoAs. As an illustrative example, at the intra-CPU level, the amount of resource sharing-and hence potential interference channels-is massive. Exhaustively identifying and bounding the timing impact of those intra-CPU interference channels is an untenable effort, given the limited information available in processor manuals. Based on our firsthand experience, this is still a challenge even in hardware simulators (e.g., GEM5 [11]). This has led timing analysis experts to prevent sharing resources at the intra-CPU RSL as a means to favor software timing analysis.

- In the automotive domain, AUTOSAR, the reference software architecture, fosters the design and deployment of reusable software components that are hardware independent. The application layer comprises software components that are further divided into entities called runnables. Despite being mapped to tasks at the AUTOSAR OS level, runnables are the UoA of software timing tools. Upon system integration, where tasks and the corresponding runnables, possibly developed by different providers, are integrated, system integrators seek ways to control the maximum timing impact that runnables can cause each other. Hence for an AUTOSAR system, the focus of software timing analysis providers should be only on the interference channels in those RSLs at which runnables can interfere with each other.

- Likewise in the avionics domain, ARINC 653 specification for space and time partitioning defines the applications (or software partitions) that can be further divided into processes. Processes can execute within a given time window or frame, either concurrently on the same core or in parallel in different cores. From the CAST-32A [7] perspective, multicore contention arises at the inter-application level. Hence, in accordance with CAST-32A guidelines, the analysis of timing interference is to be performed on those RSLs where different applications share resources. The inner RSL, instead, pertains to the WCET analysis, which is performed at the application level and is indeed subject to different requirements coming from DO-178C [12].

In the following section, we detail the different RSLs, which are also summarized in Table 2.
TABLE 2 RSL and sharing among runnables.

\begin{tabular}{|c|c|}
\hline RSL & Considerations when shared among UoA \\
\hline $\begin{array}{l}\text { Intra-CCPLEX } \\
\text { Intra-cluster }\end{array}$ & $\begin{array}{l}\text { UoAs can share resources at both RSLs. } \\
\text { Techniques exist to control the contention } \\
\text { of interference channels in these RSLs }\end{array}$ \\
\hline Intra-core & $\begin{array}{l}\text { Sharing allowed in certain CPUs. Too } \\
\text { many/complex interactions among } \\
\text { runnables to bound interference channels }\end{array}$ \\
\hline \multirow[t]{2}{*}{ Intra-GPU } & $\begin{array}{l}\text { Default option until recently in high- } \\
\text { performance GPUs }\end{array}$ \\
\hline & Still the default case in automotive GPUs \\
\hline Intra-SM & $\begin{array}{l}\text { Enabled recently with NVIDIA MPS. Not } \\
\text { yet in TX2/Xavier }\end{array}$ \\
\hline $\begin{array}{l}\text { intra-GCP } \\
\text { intra-SMB }\end{array}$ & $\begin{array}{l}\text { Not yet available. These RSLs can be } \\
\text { implemented to increase resource } \\
\text { utilization and performance. }\end{array}$ \\
\hline
\end{tabular}

\subsection{SoC CPU Component}

At the intra-cluster level, tasks can share resources. Shared caches have been identified as an issue to timing analysis [13]. However, hardware and software solutions exist to avoid that tasks evict each other's data from the cache [14] and even suffer a delay in the access to cache banks [13]. For in-cluster interconnection, usually a simple bus, the use of predictable policies such as roundrobin or FIFO, enables deriving bounds to contention [13].

For some NXP cores like the e6500 [15] used in T2080 boards [16], tasks can share resources at the intra-core level. However, how to master contention at this low-level granularity is still an open challenge, addressed by few research works [17]. Hence, in general, hyper-threading (simultaneous multithreading execution) is disabled for real-time workloads. It is also the case that NVIDIA SoCs for automotive do not support hyper-threading, hence preventing intra-CPU sharing.

\subsection{SoC GPU Component and CUDA}

Without loss of generality, we focus on NVIDIA GPUs and the terminology because of their relevance in the automotive market. However, the same analysis can be done for the software abstractions provided by other GPU vendors.

The execution of GPU programs is initiated by the CPU code (i.e., regular OS tasks) triggering CUDA operations. The latter are associated with different (one or several) CUDA streams, which determine whether they can run concurrently. We are interested in contention occurring between any GPU software entity, at any GPU abstraction level (e.g., kernel or thread), that are triggered by different OS processes (tasks) that match the UoA usually used in software timing analysis. The contention between GPU software entities triggered by the same task, e.g., different threads of the same task, relates to the timing analysis of the task in isolation. We focus, instead, on the contention effects that can arise, especially upon integration, between different units of analysis, likely to be developed by different software providers. 
For NVIDIA devices, the programmer writes a kernel and specifies the number of threads that will be used for its execution, as well as their organization in up to three dimensional blocks of threads, belonging to a grid. The thread blocks are assigned to an SM where they are executed at thread warp granularity. Each warp consists of 32 threads, and it is the minimum number of threads that are executed in parallel on the SM resources. The execution of a thread is performed by the CUDA cores in an SM (or SMB) with no specific mapping between threads and cores since their assignment takes place dynamically at run time. At a higher abstraction level, a CUDA stream is a sequence of CUDA operations (kernels and memory transfers) that execute in issue-order on the GPU. Hence, in order to increase concurrency, multiple CUDA streams can be defined by the programmer, with the CUDA operations in different streams allowed to run in parallel, provided that there are enough resources to accommodate them.

We identify the following GPU contention scenarios:

- No GPU sharing. Traditionally, GPUs have not been shared, i.e., only thread blocks of kernels belonging to the same OS process (task) could run simultaneously. This was the case before devices with Compute Capability 3.2 and CUDA v4, where support for MPS was added.

- GPU sharing. MPS allows kernels from different tasks to execute in the same GPU and, in particular, to run in parallel on different SMs. When MPS is not enabled/ supported, different tasks only time-share the GPU device. While this is the current behavior on both TX2 and Xavier, making the internal resource sharing of the GPU of no relevance, we can reasonably expect that MPS will be soon enabled to improve key metrics like resource utilization and performance efficiency. For this reason, we extend our analysis to consider also intra-GPU sharing.

- SM sharing. When MPS is enabled on Volta GPUs like Xavier, blocks from different tasks can share the same $\mathrm{SM}$, so contention between tasks arises at the intra-SM level.

- GPC and SMB sharing. To our knowledge, support for sharing resources at this RSL between blocks from different tasks is not available. Increasing resource sharing and, hence, performance could be provided in the future. As for intra-CPU sharing, in our view, interaction among UoA would be too complex to be accurately mastered.

\subsection{Intra-SoC}

This level represents the entire MPSoC and hence encompasses all compute elements implemented such as CPUs, GPUs, and DLAs.

At this level, contention can be controlled by exploiting specific software support to enforce analysis-time utilization bounds. For instance, authors in [18] and [19] propose a memory bandwidth management system to enforce memory usage quotas between multiple CPUs and multiple CPUs and an integrated GPU, respectively. In [20], instead, run-time monitoring is used to ensure that co-runner requests stay under a given utilization threshold determined at analysis time.

\section{RSL and Contention Modeling in the Jetson TX2 and Xavier}

In this section we tailor our generic RSL taxonomy to the Jetson TX2 and AGX Xavier (Section 4.1). For those resources considered relevant, we provide an early characterization of the contention UoA that can incur (Section 4.2) and show baseline models exploiting the maximum observed interference to analytically capture the effect of contention (Section 4.3).

\subsection{RSL in the $T X 2$ and $A G X$ Xavier}

Jetson TX2. Jetson TX2 implements a derivative of Parker, whose main architecture is sketched in the block diagram of Figure 2. The GPU features two SMs with four SMBs each. Each SMB comprises 32 CUDA cores for a total of 256. The CCPLEX comprises two clusters in each of which the L2 is also shared. The CPU Switch Fabric (CSF) is shared across

FIGURE 2 RSL and description of the Parker SoC as in the Jetson TX2. I\$ and D\$ stand for IL1 and DL1, respectively.

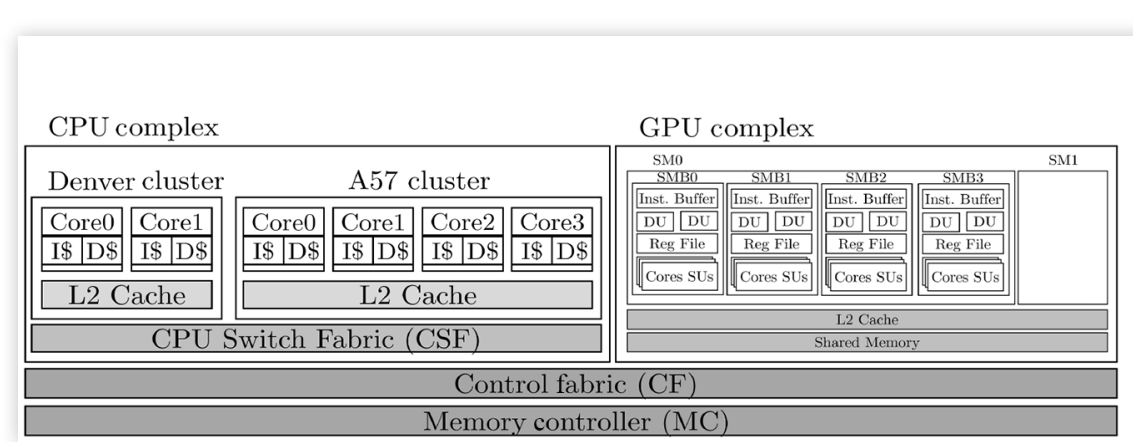


clusters. In the Denver2 cluster, there are two cores each with its private first-level instruction and data caches (referred to as IL1 and DL1, respectively). In the other cluster, there are four ARM Cortex-A57 cores also with private IL1 and DL1 caches.

In the bottom-right table of Figure 2, we describe the RSLs in the TX2: there is no intra-CPU sharing, the L2 caches are shared at the intra-cluster level, and the CSF at the intraCCPLEX level; on the GPU side, some resources are shared at the intra-SMB level and the L2 cache at the intra-GPU level. Hence, in the CCPLEX, contention between runnables can happen in all levels but intra-CPU, since cores do not support multithreading. In the GPU, SMs cannot be shared across kernels of different tasks, so only intra-GPU contention for different kernels in the L2 is relevant. Regarding software configuration, Jetson TX2 uses Jetpack 3.3, CUDA 9.2, and Linux kernel 4.4.38-tegra, and both CPUs and the GPU are operating at the highest frequency.

Jetson AGX Xavier. In the Xavier SoC (see Figure 3), the CCPLEX comprises eight homogeneous single-thread cores (no intra-CPU sharing) that are paired into four clusters and two Carmel cores per cluster. Each core has its own private IL1 and DL1, while the L2 is shared between cores in each cluster (intra-cluster sharing); hence, there are four L2s; while the L3 and the CSF are shared between all cores (intra-CCPLEX).

In the GPU, there are four GPCs (omitted for clarity in Figure 3), each with two SMs and one ROP. Each SM is subdivided into four SMBs each and includes tensor cores and Special Functional Units that are shared between threads in the SMB (intra-SMB), while SMBs in an SM (intra-SM) share the IL1, DL1, and some shared memory. Each single SMB has an instruction cache (LOI\$), a dispatch unit, warp scheduler unit, register file, and computation units. As shown in Figure 3 , Xavier has two types of computation units: regular cores (known as CUDA cores) and Tensor cores. At the intraGPU level, SMs share an L2 cache and the path to memory. Jetson AGX Xavier uses Jetpack 4.1, CUDA 10.0, and Linux kernel 4.15.0-42-generic. Both CPU cluster and the GPU are operating at the highest frequency (power mode 0 as provided by NVIDIA).

Overall, at the CCPLEX level-and similar to the TX2 case-the CPUs are single threaded, and hence there is no contention between runnables within the CCPLEX.

\subsection{Contention Characterization}

For some multicore SoCs, like the Infineon's AURIX Tri-Core, already adopted in several automotive products, some studies cover the impact on timing due to contention in hardware resources ranging from memory [21] to flash [22]. Similar trends are observed in avionics in which the multicore has been considered as a baseline computing solution for several types of systems for more than a decade [ $[\underline{3}, \underline{4}]$.

For multicore SoCs with more aggressive designs, e.g., the Renesas R Car H3 SoC [23] that includes several clusters of cores and cache levels, mastering contention remains an open challenge. GPUs are in a similar situation, with the analysis of software timing issues still in its infancy: some initial works-which we present in the related work sectionfocus on simulated GPUs, treating the GPU as a black box, analyzing low-level scheduling issues or memory contention issues $[\underline{19}, \underline{24}, \underline{25}, \underline{26}, \underline{27}, \underline{28}]$.

In this section, we perform a contention analysis of the Jetson AGX Xavier. It contains the same SoC, used in the NVIDIA DRIVE Xavier and Pegasus platforms, which are exclusively provided to certain NVIDIA partners and car manufacturers. We also analyze the previous NVIDIA Tegra Parker SoC used in the NVIDIA DRIVE PX2 platforms, which is already deployed in existing AD cars such as Tesla.

Benchmarks. We build on an incremental approach to analyze contention interference that tasks can generate on each other. The experimental framework exploits microbenchmarks specifically tailored to create contentions in different levels of memory hierarchies and represent extreme cases. Arguably, therefore, real applications will incur less contention.

Each experiment deploys one element from a small set of CPU/GPU microbenchmarks that we have programmed so that most instructions are load or store (when it matters, e.g., for write-back caches) memory operations to increase pressure in the shared resources.

- The CPU code comprises a loop with iteration load instructions with a predefined stride among them so that they access different lines in the desired cache level. Loads access a vector of the desired capacity to systematically miss in lower cache levels and hit the desired cache level occupying a big chunk of it.

FIGURE 3 RSL and description of the Xavier SoC implemented in Jetson Xavier.

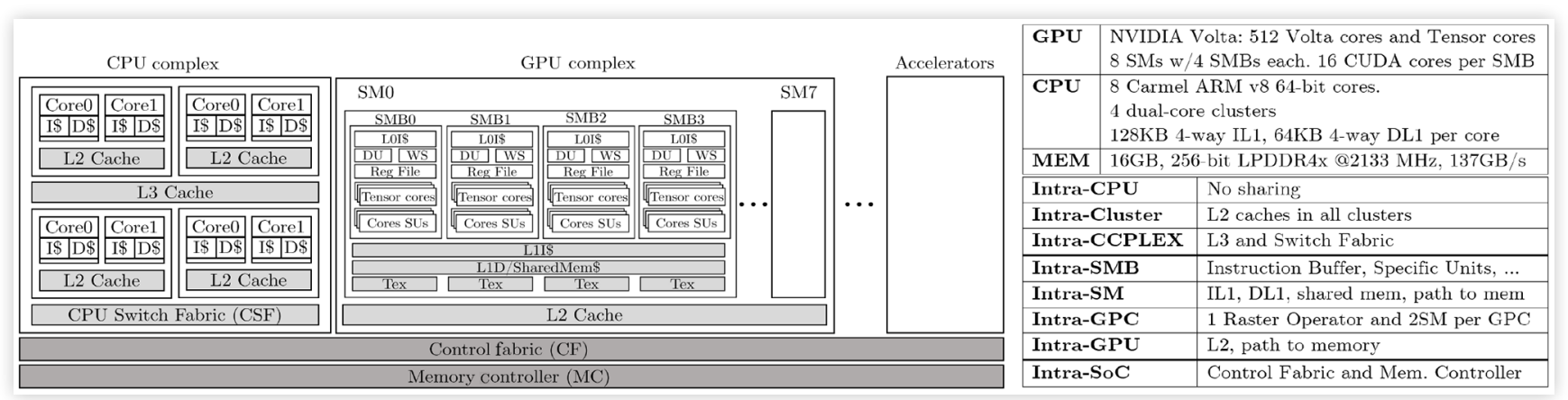

(c) Hamid Tabani, Leonidas Kosmidis, Enrico Mezzetti, Jaume Abella, Francisco J. Cazorla. 
- The GPU code comprises a kernel with a loop with iteration load instructions with a predefined and random stride among them so that they access different lines in the desired cache level. By launching the kernel, hundreds of threads are instantiated to load a vector of the desired capacity (e.g., L2 cache or mem) to systematically miss in lower cache levels and hit the desired cache level occupying a big chunk of it.

- The NVDLA code comprises a representative deep neural network (DNN) configuration, which performs inference and loads gigabytes of data per second from memory. Unlike the GPU and CPU codes, NVDLA is designed mainly for performing deep learning inference; therefore, we configured it to launch and perform an object detection DNN.

We exercise enough control to make a microbenchmark hit/miss in the desired cache level (L1hit, L2hit, L3hit, mem). The data footprint of the benchmarks occupies a big share of the cache level they access, so when we run several copies of the same benchmark, the vast majority of the hits that the benchmark experiences when runs in isolation become misses. Also, when a benchmark hits a cache level $\left(L_{x}\right)$, it misses the lower cache levels $L_{x-1}, L_{x-2}, \ldots$.

- L1hit. A benchmark in which more than $95 \%$ of the instructions are loads/stores that hit in the $\mathrm{L} 1$ cache.

- L2hit (i.e., L1miss). A benchmark in which more than $95 \%$ of the instructions are memory operations that miss in the L1 cache and hit in the L2. It occupies a larger part of the L2.

- L3hit (L1miss and L2miss). Similar to L2hit but missing in L1 and L2 and hitting in L3.

- Mem. A benchmark for which most of its instructions are memory operations that miss in all caches.

Scenarios. To describe each test scenario we adopt the following conventions:

- In TX2, each experiment is defined as $[x x x x ; x x][y y]$, where " $\mathrm{x}$ " is a placeholder for a task (benchmark) we run in the CCPLEX, either in the four cores of the A57 (before the ";") or the two cores in the Denver2 (after the “;”). " $y$ " is a placeholder for a kernel that can be run in any of the SMs in the GPU.

- Tests are defined as $[x x ; x x ; x x ; x x][y y y y y y y y][z]$ for Xavier, describing the eight tasks (x) that can run in pairs (separated by “;") in the CPU, the eight kernels (y) that can run in the SMs of the GPU, and the software that can run in the NVDLA (z).

In all test instances, $\mathrm{x}, \mathrm{y}$, and $\mathrm{z}$ can either be the analysis task (referred to as A or AT) on which we track the impact of contention, be a contender task (referred to as C or CT), or be left empty (“" or “-”).

In our experiments, all instances of the CT are of the same type, i.e., L1hit, L2hit, L3hit, or mem. Results with mixed (heterogeneous) instances offer no higher slowdown than what we report below for homogeneous CTs.

\section{FIGURE 4 TX2 results: CCPLEX.}

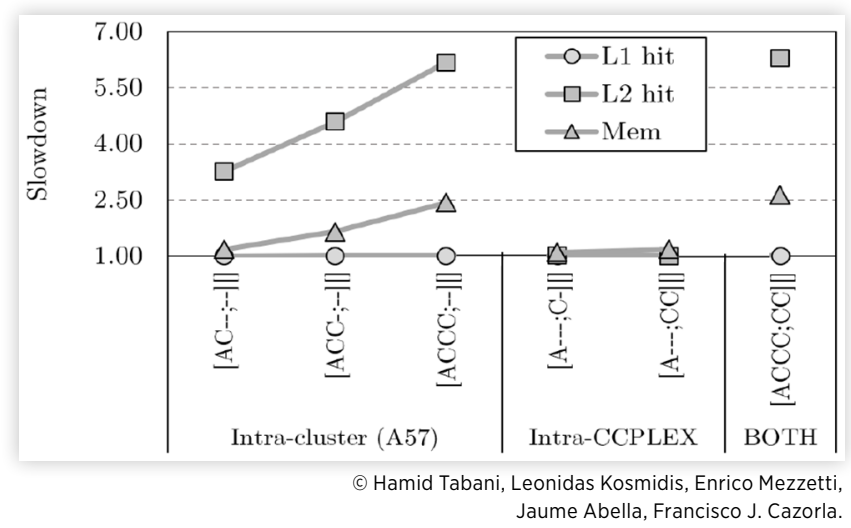

Metric. In our experiments, we analyze the slowdown the AT suffers when it runs with other CTs w.r.t. the case when it runs in isolation. It is defined as $S_{A T}=E T_{A T}^{i s s l} / E T_{A T}^{m u c}$. When the slowdown the AT suffers is below the core count, then we say that the MPSoC provides performance benefits. Instead, if the AT slowdown is above the core count, it is better to run the AT and the CTs in isolation and serially, i.e., one after the other.

4.2.1. Jetson TX2 A57 Intra-cluster. In Figure 4 (left) for the A57 intra-cluster results, we observe the following:

- When AT hits in L1 (L1hit) (circles in Figure 4), it is insensitive to the contention created by CTs in the other cores, regardless of the level in which CTs hit/miss.

- When the AT and its CTs hit in the L2 (squares in Figure4), contention significantly slows down the AT. This is so since most AT L2 hits become misses due to $\mathrm{CT}$ evictions, and the AT further suffers contention with CT accessing memory.

- When the AT/CT are memory bound, AT suffers lower relative contention (below $2.5 \times$ ) since its memory operations in isolation miss in the L2 anyway, so under a multicore setup AT misses just suffer an extra delay in accessing memory.

When the AT and CT are L2hit, they suffer slowdown above the core count (4), which suggests that executing sequentially the tasks will result in a higher performance. For the other cases (L1hit and mem), slowdowns are well below $4 \times$, resulting in performance improvement when several tasks run in parallel.

Intra-CCPLEX. Figure 4 also shows that when the AT (and the CT) are mapped to L1hit and L2hit, they suffer no inter-cluster (i.e., intra-CCPLEX) contention. When both AT and the CTs constantly access the main memory, AT suffers a small slowdown of $17 \%$.

Intra-cluster and Intra-CCPLEX. Finally, when the AT suffers contention from CT in the A57 cluster and the Denver2 cluster, its slowdown slightly increases w.r.t. the intracluster results.

Intra-GPU. Figure 5 reports the impact of running two kernels (one AT and one CT) of the same type in the SMs so that both are either L2hit or memory bound. Recall that the TX2 features only two SMs. The observed intra-GPU 
FIGURE 5 TX2 results: GPU.

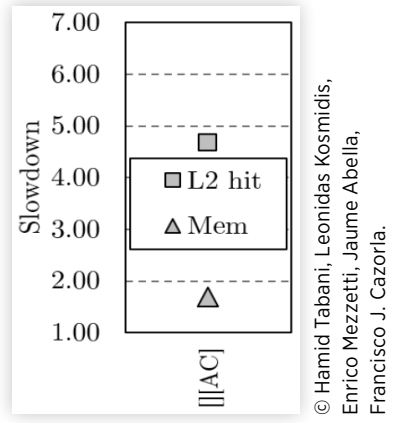

slowdown for the memory-bound case is high $(1.68 \times)$ but still below the SM count. Things change radically in the L2hit scenario, with both kernels hitting in the L2, where contention leads to a $4.68 \times$ slowdown, hence, quite above the SM count. This calls for mechanisms to control contention in L2 or to prevent L2-cache-bound kernels from sharing the GPU.

Intra-SoC. Figure 6 reports the maximum slowdown the AT suffers when running in the CPU and CTs run in the GPU, and vice versa (scenarios $C P U \rightarrow G P U$ and $G P U \rightarrow C P U$, respectively). We observe that the AT suffers a $5 \times$ slowdown when running in the GPU and CTs run in the CPU (scenarios CPU $\rightarrow G P U$ ). However, in the GPU $\rightarrow C P U$ scenario, the observed slowdown is relatively small, up to $26 \%$. The last two scenarios in Figure 6 show the slowdown when the AT runs in the GPU and $\mathrm{CPU}$, respectively, and suffer the maximum contention from all CPU cores and GPU SMs, respectively. Comparing the results with those of Figures 4 and $\underline{5}$, we observe that most of the contention happens at the intra-CCPLEX or intra-GPU levels. For instance, comparing scenarios []$[A, C]$ and $[C, C$, $C, C][A, C]$, the slowdown goes from 1.68 (mem) and 4.68 (L2hit) to 2.06 (mem) and 5.74 (L2hit).

\section{FIGURE 6 Slowdown at the SoC level (TX2).}

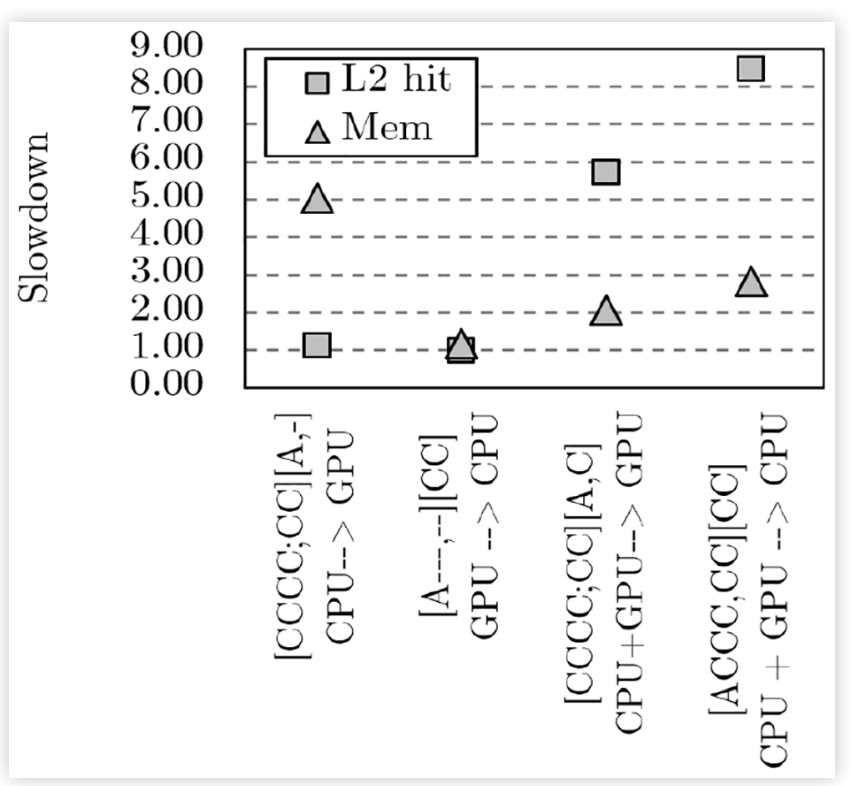

(c) Hamid Tabani, Leonidas Kosmidis, Enrico Mezzetti, Jaume Abella, Francisco J. Cazorla.
4.2.2. Jetson AGX Xavier In this section, we omit the intra-GPC contention results since the ROP, shared at this level, is not used for general-purpose computation.

Intra-Cluster. Unlike TX2, the Xavier CCPLEX comprises homogeneous clusters, which simplifies intracluster analysis. In the setup [A,C;-,-;-,-;-,-][][] the AT and the CT run on the same cluster, so they share intra-cluster, intraCCPLEX, and intra-SoC resources. At intra-cluster level, Figure 7, we observe an increasing slowdown as the CT and AT access upper cache levels: from no slowdown when they access L1 (since it is private per core) to $1.26 \times, 1.50 \times$, and $1.82 \times$ when they access L2, L3, and memory, respectively. In the latter case, slowdown tightly matches the number of cores in the cluster, so it matches the maximum allowed slowdown that justifies using a multicore.

Intra-CCPLEX. In these experiments we analyze the impact on $\mathrm{AT}$ when an increasing number of CT runs on different clusters of the CCPLEX, i.e., setups ([A,-;X,X;X,X;X,X] [][]), see Figure 7. In all setups there is no slowdown when AT/ CT are L1hit and L2hits since each cluster has its own private $\mathrm{L} 1$ and $\mathrm{L} 2$. When AT/CT are L3 and mem, the contention AT suffers increases almost linearly w.r.t. the number of CTs. Yet contention does not exceed $1.55 \times$ and $1.2 \times$ for the L3 and memory-bound cases, respectively, when there are 6 CTs running in other clusters.

The last setup $[A, C ; C, \mathrm{C} ; C, \mathrm{C} ; C, C][][]$ covers both intra-cluster and intra-CCPLEX slowdown. Even in this extreme scenario, the observed slowdown is only $1.31 \times, 1.83 \times$, and $2.21 \times$.

Intra-GPU. For the GPU we use L2hit and mem benchmarks. Figure 8 reports the slowdown one of these benchmarks (AT) suffers as we run an increasing number of copies of the same benchmark (CT). When the AT-CT are memory bound, the AT does not suffer extra L2 misses but just experiences extra latency per miss, which slows it up to $1.4 \times$ when

\section{FIGURE 7 Xavier: Intra-CCPLEX.}

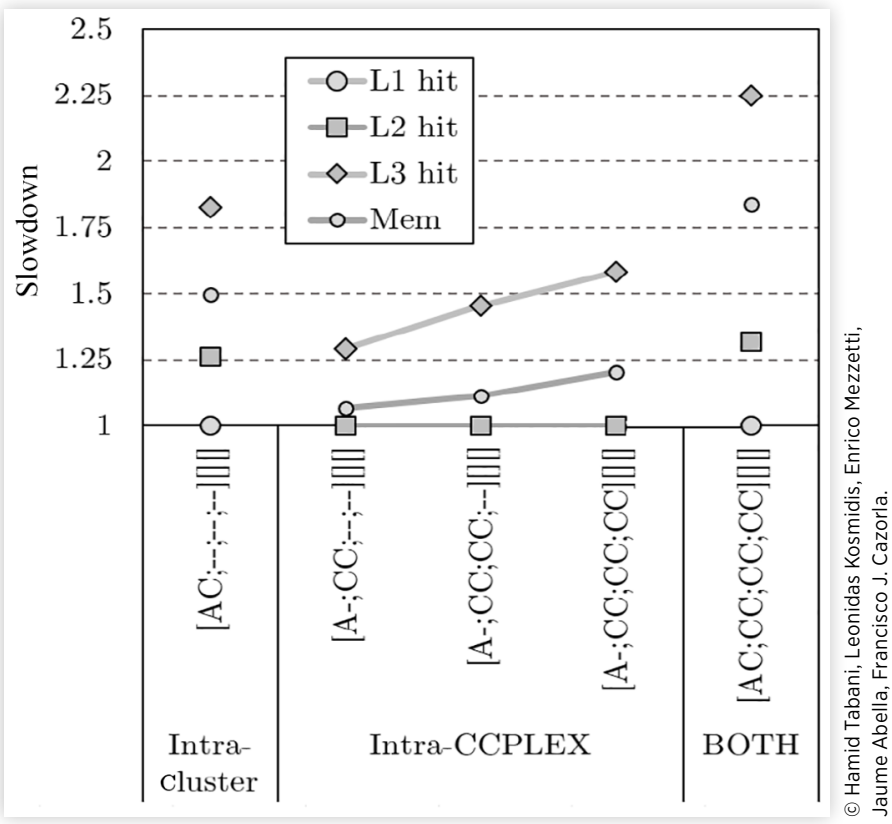


FIGURE 8 Xavier: Intra-GPU.

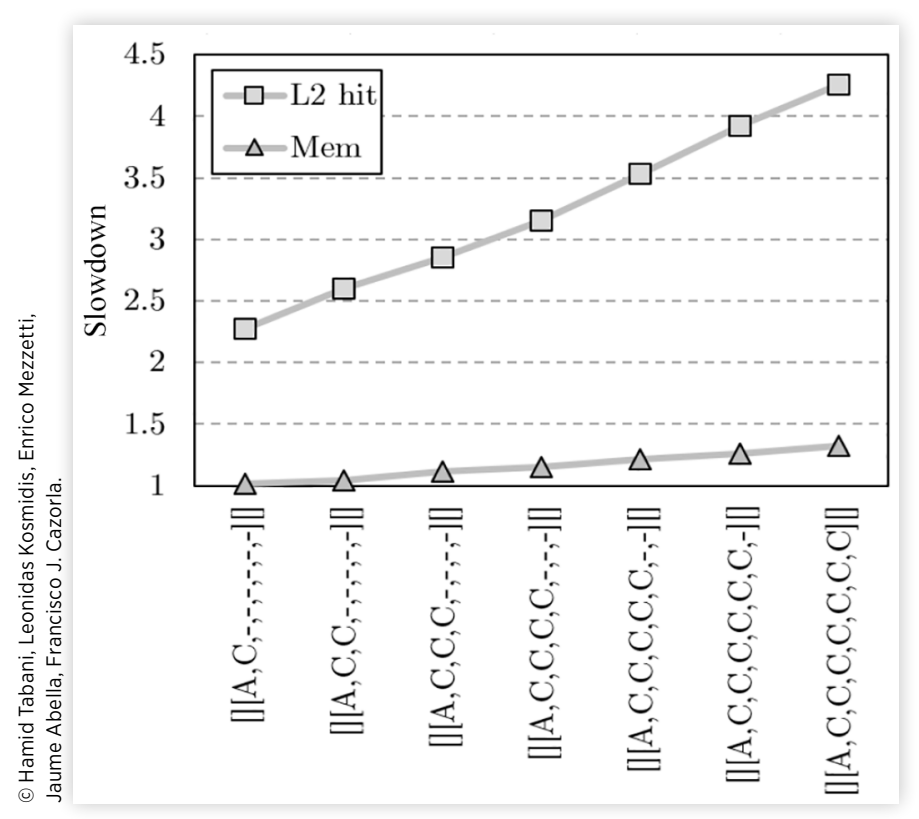

there are seven CTs. When the AT-CT are L2 bound, CT causes extra misses in the AT, resulting in a higher impact on its behavior. Despite the maximum observed slowdown is $4.26 \times$, it is moderate since there are eight memory-intensive and highly sensitive kernels running in parallel.

Intra-SoC. Figure 9 presents an in-depth analysis of the impact of running AT in one computing element (i.e., CPU, GPU, or NVDLA) and the CTs in one or the other two computing elements.

When CTs run in the GPU, i.e., GPU $\rightarrow C P U$ and $G P U \rightarrow$ $D L A$, they generate a slowdown of up to $1.6 \times$ and $2.4 \times$ on the AT when it runs in the GPU and the NVDLA, respectively. In both cases the slowdown increases quite linearly from one to four CTs. At that point the impact of CTs on the AT saturates and remains roughly unchanged up to eight CTs. This is so because four CTs fully occupy the memory bandwidth, causing the maximum contention on the AT.

When CTs run in the CPU, i.e., $C P U \rightarrow G P U$ and $C P U \rightarrow$ $D L A$, they generate a lower slowdown on the AT than when they are running on the GPU or DLA. In both cases, the slowdown increases with the number of CTs and never goes beyond $1.2 \times$.

When the CT runs in the DLA, it generates more contention compared to the case when the AT runs in the GPU.

Combined effects, e.g., $G P U+C P U \rightarrow D L A$, are shown in Figure 9 (right). We have observed that the main reason why the AT, when run in the CPU, suffers less slowdown in comparison to when it runs in the GPU is that the CPU AT generates relatively fewer memory requests than the GPU AT. Therefore, although considerable, the CPU AT faces less contention.

4.2.3. Discussion The potential contention happening at a given RSL depends on the amount of parallelism allowed by the target architecture. Contention at each RSL is only acceptable as long as it can be controlled (i.e., contained) to limit the inter-task contention slowdown, thus avoiding timing faults []ㅡ.

A full in-depth analysis of the source of contention in the TX2 and AGX Xavier requires more experimentation resulting in a technical report well beyond the size of this paper. However, the characterization of the contention in the different RSLs we assembled in this section provides a solid baseline to accomplish safety standard requirements in terms of bounding interference channels []] and provide FFI []].

From the results obtained, it is clear that the complex nature of multicore GPU-based SoCs prevents achieving FFI on task execution time. Under this scenario, it is acceptable to argue on FFI by applying the same concept to the bounds derived on task execution time, so that they remain valid under any possible interference the task can suffer. For both the TX2 and the AGX Xavier, the cache level shared on the CCPLEX and the GPU are the main hardware block affecting FFI. For the A57 cluster in the TX2, contention impact goes beyond the core count (4) and in the CPU clusters in the Xavier nearly reaches it (2). In both SoCs we observe a significant slowdown between SMs at the intra-GPU level due to L2 sharing that goes up to $4.68 \times$ (TX2) and 4.26× (Xavier). It follows that hardware and software cache partition solutions are required to control contention in these caches, without imposing overly pessimistic bounds on timing.

\section{FIGURE 9 Xavier: Intra-SoC.}

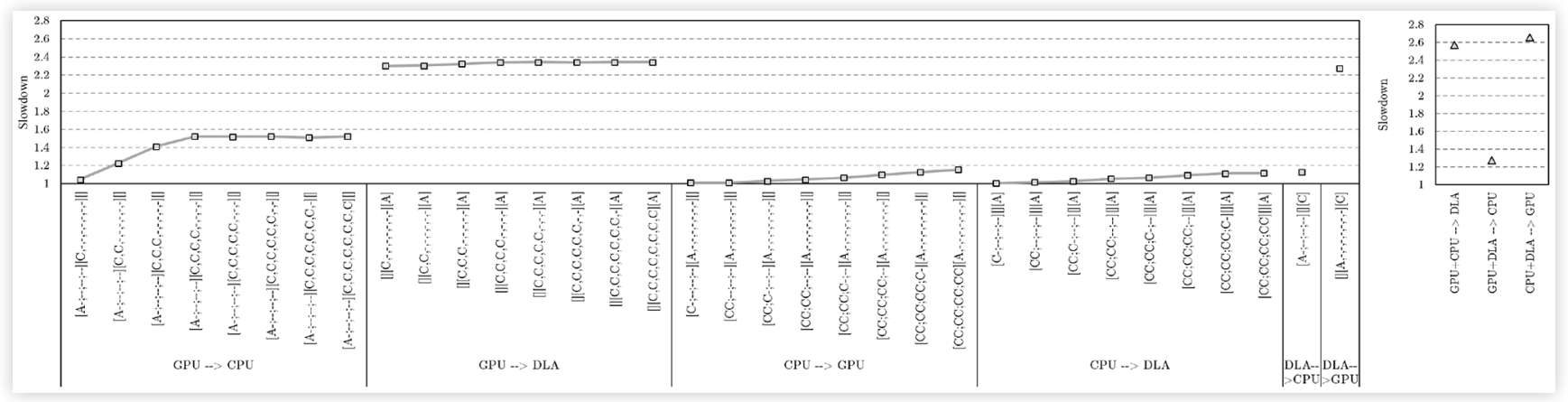




\subsection{Early-Stage Contention Modeling}

As a result of the extensive empirical effort, we obtained an early characterization of the timing interference on the Jetson Xavier and TX2 platforms. Such characterization is intended to allow a preliminary assessment of the contention effects a given application may potentially suffer on those platforms and to steer the in-deep analysis effort.

As a general observation, we note that, except for the case of high utilization of shared caches (high hit rate on L2/L3), contention impact in the considered platforms is reasonably low despite the aggressiveness of the evaluated workloads. We conclude that both the Jetson Xavier and the TX2 offer a promising solution to increase parallelism in automotive SoCs, i.e., running more parallel tasks, while increasing overall guaranteed performance. For cache-intensive applications, taking a large benefit from shared caches, countermeasures need to be put in place in order to minimize and control the amount of interference. These countermeasures may include cache partitioning in the CCPLEX and contentionaware scheduling strategies for the GPU Complex.

From the characterization, we also observe that contention is almost linear with the number of CTs. This significantly simplifies analytical modeling of contention effects-in addition to facilitating in providing an intuitive argument for certification. As an example, Table 3 describes a simple linear model for deriving early estimates on the maximum contention slowdown $(m c s d)$, relative to the application execution time, for the TX2 intra-cluster contention and AGX Xavier intra-SM contention. The formulas, which are linear on the number of contending tasks/kernels $N_{C T}$, build on the empirical characterization results discussed in the previous subsections and are to be applied depending on the application characteristics (whether L2 or memory intensive). Note that $N_{C T} \geq 1$ is assumed in the models so that, in case of no CTs, mcsd equals 1 . As already observed, this baseline model is not intended to provide an upper bound to the contention effects but is to be interpreted as an early indication of the potential impact of running an application that is either L2 intensive or memory intensive on the target MPSoC.

\subsection{Performance Improvements}

In previous sections, we have focused on contention. In particular, we cover high-contention scenarios to capture the requirements in terms of predictability and software timing

TABLE 3 Example of linear contention models.

\begin{tabular}{|c|c|c|}
\hline RSL & AT/CT type & Linear model \\
\hline TX2. I-Cluster & L2hit & $\mathrm{mcsd}=3.25+\left(\mathrm{N}_{\mathrm{CT}}-1\right) \times 1.47$ \\
\hline TX2. I-Cluster & Mem & $\operatorname{mcsd}=1.17+\left(\mathrm{N}_{C T}-\mathrm{I}\right) \times 0.48$ \\
\hline Xavier. I-SM & L2hit & $\operatorname{mcsd}=2.30+\left(\mathrm{N}_{\mathrm{CT}}-1\right) \times 0.33$ \\
\hline Xavier. I-SM & Mem & $\operatorname{mcsd}=1.02+\left(\mathrm{N}_{\mathrm{CT}}-1\right) \times 0.08$ \\
\hline
\end{tabular}

FIGURE 10 CPU performance improvement when the number of cores for the computation is increasing.

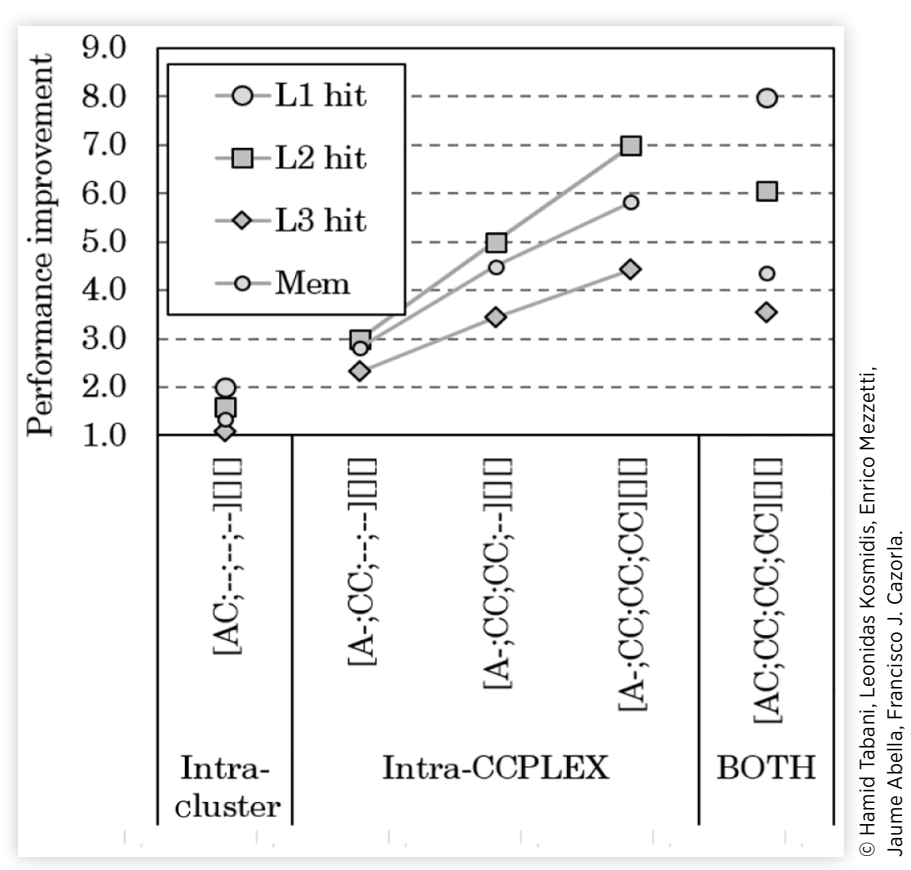

verification of safety standards like CAST-32A and ISO 26262. Our contention analysis has been performed from the MPSoC level, which we have referred to as Intra-SoC, down to the individual computing elements (e.g., intra-CPU and intra-GPU).

The particular performance improvement that can be achieved in MPSoCs is application dependent. This relates to the ratio of computations (performed locally in the core) w.r.t. memory accesses (that required accessing shared resource where contention arises) in a given application. The results we have collected, in terms of contention, can help understand the potential performance improvements.

Figure 10 compares the execution time it takes eight copies of the L1hit, L2hit, L3hit, and mem benchmark to execute when run in parallel using an increasing number of cores versus the time it takes them to execute serially, i.e., using one core.

- As we can see, in those scenarios in which the application resides mainly in the L1 cache, the performance improvement (big circle) is linear with the number of cores.

- If it resides in L2 (squares), we see less than ideal performance improvement for the intra-cluster case as the L 2 is shared among both cores achieving a performance improvement of $1.3 \times$. For the intraCCPLEX scenarios, the performance improvement is linear with the number of cores (note that squares hide big circles for these 3 scenarios). When all eight cores are active, the performance improvement is $6 \times$.

- As expected, the performance improvement reduces for L3 and mem benchmarks as they share some resources that make their performance not increase linearly. 
FIGURE 11 GPU performance improvement when the number of SMs used for the computation is increasing.

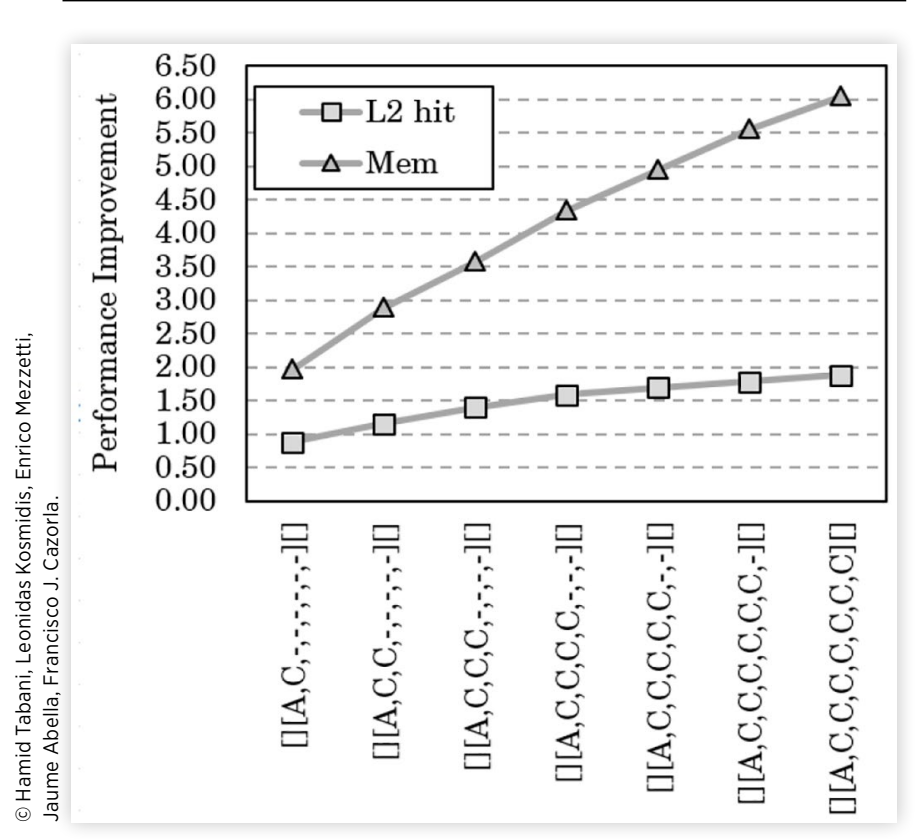

Likewise, Figure 11 makes a similar comparison for the GPU. In this case, we see that as we increase the number of SMs used for the computation, the performed L2 accesses that happen at the same time start missing, limiting the performance improvement, as it can be seen from the small slope of the line. On the other hand, if the GPU operations are mainly targeting memory, we can see that the performance scalability is higher since the memory accesses suffer less as they already miss in isolation.

In addition to the same resource performance improvement we have presented so far, in order to provide a complete application view, we have analyzed a number of scenarios where a varying number of tasks are deployed in the different computing units. Those include from one to eight tasks in the cores, from one to eight tasks in the GPU, and up to one task in the DLA. For those scenarios, we consider three different task deployments:
- Serial. All tasks are run serially, as in a single-core SoC, and thus experiencing no contention.

- Parallel 100\%. All tasks are run in parallel, namely, one CPU task per core, and all GPU tasks running in parallel in the GPU. The tasks chosen are those maximizing interference, so this scenario corresponds to the worst interference one.

- Parallel 25\%. This deployment is identical to the previous one, but interference generated by the tasks is $25 \%$ that of the worst (100\%) case.

Results for this analysis are shown in Figure 12. Execution time is normalized to arbitrary time units where each task takes one time unit when run without contention. Thus the execution time for the serial scenario matches the number of tasks deployed. Execution times for the parallel cases (blue and orange columns in the plot) show that, naturally, execution time decreases by exploiting the computing resources of the MPSoC running all tasks simultaneously. The plot shows execution times in the form of columns whose scale can be found in the left $y$-axis and performance boosts in the form of dashed lines whose scale can be found in the right $y$-axis.

High-Interference Scenarios. For the parallel 100\% scenario, i.e., the scenario maximizing contention, we observe the following trends.

- As shown, in the leftmost group of columns (8 GPU group), increasing the number of CPU tasks leads to slightly increasing the execution times since interference caused and experienced by CPUs is limited.

- Instead, the central-right group of columns (8 CPU group) shows that increasing the number of GPU tasks leads to a non-negligible increase in the execution time since tasks in the GPU are less isolated than those in the cores and, hence, cause each other higher interference. This effect mostly relates to the fact that cores have limited bandwidth to access shared caches and memory, thus not saturating it by themselves; whereas the GPU has high shared cache and memory bandwidth, which allows large interference across GPU tasks by saturating the bandwidth.

\section{FIGURE 12 Execution time and performance boost for different scenarios varying the number of CPU, GPU, and DLA tasks.}

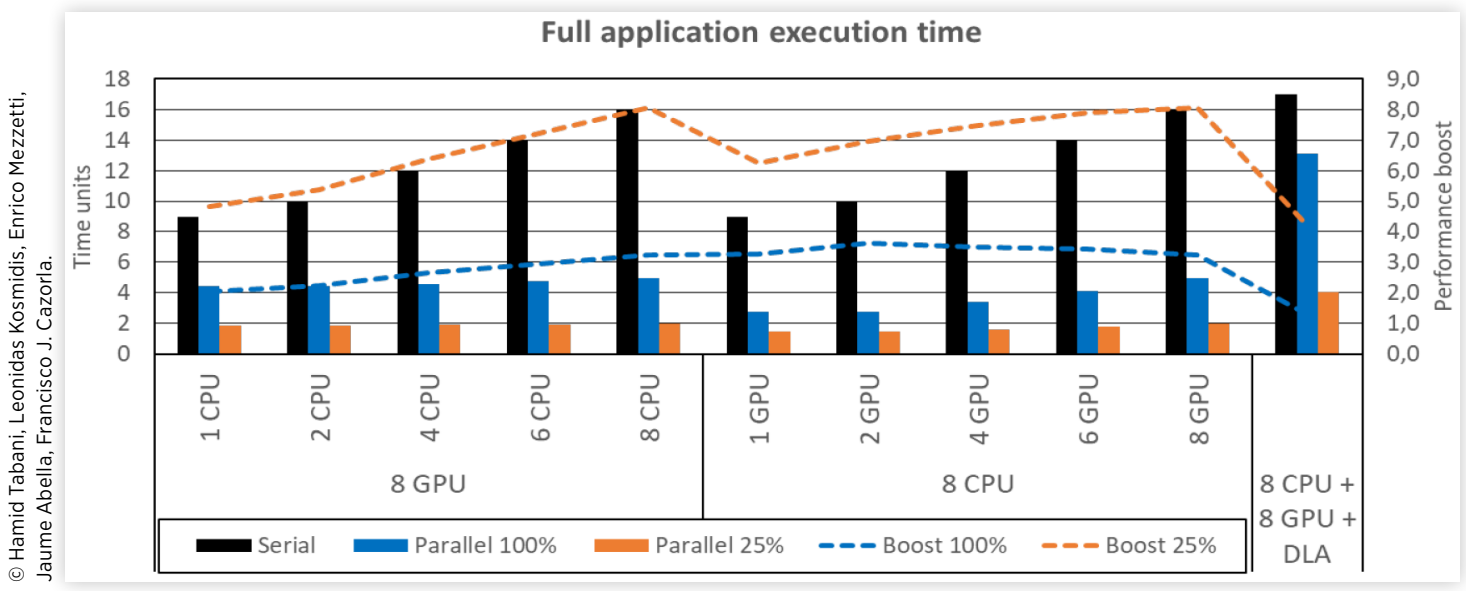


These trends are better observed looking at the dashed lines that report the performance boost w.r.t. the serial case. As shown for the parallel $100 \%$ case, increasing the number of $\mathrm{CPU}$ tasks allows increasing the performance boost from $2 \times$ to $3.5 \times$. Instead, increasing the number of GPU tasks keeps the performance boost quite stable around 3.5x. If we further add a DLA task, memory traffic completely saturates, and the performance boost w.r.t. the serial case is only $30 \%$. Hence, if tasks produce high contention, it is ill-advised to run DLA tasks along with CPU and GPU ones. On the other hand, adding CPU tasks allows further increasing the performance obtained.

Low-Interference Scenarios. When considering lessdemanding tasks (parallel 25\%), execution times drop and never double for any individual task w.r.t. the isolation case as long as no DLA task is run in parallel. This leads to a huge performance boost that increases when also increasing the number of CPU and/or GPU tasks. The highest performance boost (above $8 \times$ ) is reached when eight CPU and eight GPU tasks are run in parallel since none of them doubles its execution time. In particular, the serial case requires 16 time units to execute all tasks, whereas the parallel $25 \%$ case requires 1.98 time units to complete the slowest task. Note that if the DLA task is also run in parallel with CPU and GPU ones, the performance boost drops down to $4 \times$ only.

Overall, we see that, for a variety of loads, integrating several tasks in the CPUs and the GPU results in performance gains when interference generated is low. Instead, DLA tasks can threaten performance gains by being highly memory bandwidth-demanding. For high-contention scenarios, building on the contention results in Section 4.2.2, the expected performance benefits reduce.

\section{Related Work}

For GPU, several works cover static timing analysis $[\underline{25}, \underline{24}]$ and measurement-based techniques [26]. The former has the limitation that considers the execution of a kernel only on a single Stream Multiprocessor or works on a simulation environment; whereas the latter treats the GPU as a black box and does not analyze contention interference, the main focus of this paper. In [10], authors characterize memory contention between CPU and GPU in NVIDIA's Jetson TK1 and TX1. We provide a comprehensive taxonomy of RSLs, analyzing the impact on intra-CCPLEX and intra-GPU contention and showing intraSoC (memory contention) effects for the TX2 and the AGX Xavier. Furthermore, both CCPLEX and the GPU architecture of the SoCs studied in this paper are considerably more complex.

Another set of works focuses on scheduling issues that arise when certain kernels are runnable but blocked, i.e., not actually running in the GPU. Unlike those works, we focus on hardware contention effects and make sure that we measure only the time kernels that are actually running. Both contention and scheduling effects need to be mastered to provide time predictability. In this line, authors in [28] reverse engineered the GPU scheduling policies on the TX2 platform, concluding that the GPU scheduler has predictable properties but sharing that the GPU among tasks from different address spaces can negatively impact predictability. In [27], authors identified mismatches between documentation and the actual behavior of the TX2 system of NVIDIA used in the automotive sector, and the MPS in desktop GPU systems.

Authors in [19] reported a maximum of 3.3x slowdown for a memory-sensitive GPU benchmark due to the contenders running in CPU cores. Likewise, we have observed that our microbenchmarks are able to create higher contention (more than $5 \times$ ).

\section{Conclusion}

Because of their inherent performance requirement, many software-provided functionalities are deployed on complex MPSoCs with GPU support. Despite MPSoCs arguably provide the performance required to sustain such functionalities, MPSoCs also expose software to potentially huge inter-core timing interference arising from contention on shared resources. Contention effects make it much more difficult to consolidate the necessary timing guarantees especially with the steady increase in the number and complexity of shared hardware.

We contend that the identification of the RSLs and their relation to the UoA are necessary steps to structure the challenge of providing timing analysis for MPSoCs, as well as reduce its costs by focusing on those RSLs where the UoA can contend. This is accompanied by early figures on the impact of contention to guide the system design and development in view of optimizing the timing analysis effort and achieving compliance to safety standards. The RSL analysis and contention results presented for the NVIDIA Jetson TX2 and AGX Xavier are used to illustrate the applicability of the presented approach.

\section{Acknowledgments}

This work has been partially supported by the Spanish Ministry of Science and Innovation under grants PID2019107255GB and FJCI-2017-34095; and the European Union's Horizon 2020 research and innovation programme under grant agreement No. 878752 (MASTECS) and the European Research Council (ERC) grant agreement No. 772773 (SuPerCom).

\section{Contact Information}

Francisco J. Cazorla (Barcelona Supercomputing Center and Maspatechnologies S.L.)

francisco.cazorla@bsc.es

francisco.cazorla@maspatechnologies.com 


\section{References}

1. ARM, “ARM Expects Vehicle Compute Performance to Increase 100x in Next Decade," 2015.

2. Koon, J., “Avionics Supercomputers of the Future,” 2018, http://interactive.aviationtoday.com/avionicssupercomputers-of-the-future/.

3. European Aviation Safety Agency (EASA), "MULCORS-Use of MULticore proCessORs in Airborne Systems," Tech. Rep., EASA.2011/6, 2011.

4. Radack, D., Tiedeman, H.G., and Parkinson, P., "Civil Certification of Multi-Core Processing Systems in Commercial Avionics," Tech. Rep., Rockwell Collins and Wind River, 2018.

5. Notebaert, O., “On-Board Payload Data Processing Requirements and Technology Trends," in European Workshop on On-Board Data Processing (OBDP2019), European Space Research and Technology Centre (ESTEC), Noordwijk, The Netherlands, 2009.

6. Wilhelm, R. and Reineke, J., "Embedded Systems: Many Cores Many Problems," in 7th IEEE International Symposium on Industrial Embedded Systems (SIES'12), Karlsruhe, Germany, 176-180, 2012.

7. Certification Authorities Software Team, "Multi-core Processors-Position Paper,” Tech. Rep., CAST-32A, November 2016.

8. International Organization for Standardization, "ISO/DIS 26262. Road Vehicles-Functional Safety," 2009.

9. Fernández, M., Gioiosa, R., Quiñones, E., Fossati, L. et al., "Assessing the Suitability of the NGMP Multi-Core Processor in the Space Domain," in Proceedings of the 12th International Conference on Embedded Software, EMSOFT 2012, part of the Eighth Embedded, Systems Week, ESWeek 2012, Tampere, Finland, October 7-12, 2012 (Jerraya, A., Carloni, L.P., Maraninchi, F.F., and Regehr, J., eds.), 175-184, ACM, 2012.

10. Cavicchioli, R., Capodieci, N., and Bertogna, M., "Memory Interference Characterization between CPU Cores and Integrated GPUs in Mixed-Criticality Platforms," in 22nd IEEE International Conference on Emerging Technologies and Factory Automation, ETFA 2017, Limassol, Cyprus, September 12-15, 2017, 1-10, IEEE, 2017.

11. Binkert, N.L., Beckmann, B.M., Black, G., Reinhardt, S.K. et al., “The gem5 Simulator," SIGARCH Comput. Archit. News 39, no. 2 (2011): 1-7.

12. Radio Technical Commission for Aeronautics (RTCA), "DO178C, Software Considerations in Airborne Systems and Equipment Certification," 2011.

13. Paolieri, M., Quinones, E., Cazorla, F.J., Bernat, G. et al., "Hardware Support for WCET Analysis of Hard Real-Time Multicore Systems," in 36th International Symposium on Computer Architecture (ISCA 2009), Austin, TX, June 20-24, 2009 (Keckler, S.W. and Barroso, L.A., eds.), 57-68, ACM, 2009.
14. Kim, S., Chandra, D., and Solihin, Y., "Fair Cache Sharing and Partitioning in a Chip Multiprocessor Architecture," in 13th International Conference on Parallel Architectures and Compilation Techniques (PACT 2004), Antibes Juan-les-Pins, France, September 29-October 3, 2004, 111-122, IEEE Computer Society, 2004.

15. NXP, “e6500 Core Reference Manual,” 2016.

16. NXP, "QorIQ T2080 and T2081 Multicore Communications Processors," 2017.

17. Paolieri, M., Mische, J., Metzlaff, S., Gerdes, M. et al., "A Hard Real-Time Capable Multi-Core SMT Processor," ACM Trans. Embed. Comput. Syst. 12, no. 3 (2013): 79:1-79:26.

18. Yun, H., Yao, G., Pellizzoni, R., Caccamo, M. et al., "Memguard: Memory Bandwidth Reservation System for Efficient Performance Isolation in Multi-core Platforms," in 19th IEEE Real-Time and Embedded Technology and Applications Symposium, RTAS 2013, Philadelphia, PA, April 9-11, 2013, 55-64, IEEE Computer Society, 2013.

19. Ali, W. and Yun, H., "Protecting Real-Time GPU Kernels on Integrated CPU-GPU SOC Platforms," in 30th Euromicro Conference on Real-Time Systems, ECRTS 2018, Barcelona, Spain, July 3-6, 2018 (Altmeyer, S., ed.), vol. 106 of LIPIcs, 19:1-19:22, Schloss Dagstuhl - Leibniz-Zentrum fur Informatik, 2018.

20. Nowotsch, J., Paulitsch, M., Buhler, D., Theiling, H. et al., "Multi-Core Interference-Sensitive WCET Analysis Leveraging Runtime Resource Capacity Enforcement," in 26th Euromicro Conference on Real-Time Systems, ECRTS 2014, Madrid, Spain, July 8-11, 2014, 109-118, IEEE Computer Society, 2014.

21. Biondi, A. and Natale, M.D., "Achieving Predictable Multicore Execution of Automotive Applications Using the LET Paradigm," in IEEE Real-Time and Embedded Technology and Applications Symposium, RTAS 2018, Porto, Portugal, April 11-13, 2018 (Pellizzoni, R., ed.), 240-250, IEEE Computer Society, 2018.

22. Li, L. and Mayer, A., "Trace-Based Analysis Methodology of Program Flash Contention in Embedded Multicore Systems," in 2016 Design, Automation \& Test in Europe Conference \& Exhibition, DATE 2016, Dresden, Germany, March 14-18, 2016 (Fanucci, L. and Teich, J., eds.), 199-204, IEEE, 2016.

23. RENESAS, “RENESAS R-Car H3,” 2016, https://www. renesas.com/kr/en/solutions/automotive/soc/r-car-h3.html.

24. Betts, A. and Donaldson, A.F., "Estimating the WCET of GPU-Accelerated Applications Using Hybrid Analysis," in 25th Euromicro Conference on Real-Time Systems, ECRTS 2013, Paris, France, July 9-12, 2013, 193-202, IEEE Computer Society, 2013.

25. Berezovskyi, K., Bletsas, K., and Andersson, B., "Makespan Computation for GPU thReads Running on a Single Streaming Multiprocessor," in 24th Euromicro Conference on Real-Time Systems, ECRTS 2012, Pisa, Italy, July 11-13, 2012 (Davis, R., ed.), 277-286, IEEE Computer Society, 2012. 
26. Berezovskyi, K., Guet, F., Santinelli, L., Bletsas, K. et al., "Measurement-Based Probabilistic Timing Analysis for Graphics Processor Units," in Architecture of Computing Systems-ARCS 2016-29th International Conference, Nuremberg, Germany, April 4-7, 2016, Proceedings (Hannig, F., Cardoso, J.M.P., Pionteck, T., Fey, D. et al., eds.), vol. 9637 of Lecture Notes in Computer Science, 223-236, Springer, 2016.

27. Yang, M., Otterness, N., Amert, T., Bakita, J. et al., “Avoiding Pitfalls When Using NVIDIA GPUs for Real-
Time Tasks in Autonomous Systems," in 30th Euromicro Conference on Real-Time Systems, ECRTS 2018, Barcelona, Spain, July 3-6, 2018 (Altmeyer S., ed.), vol. 106 of LIPIcs, 20:1-20:21, Schloss Dagstuhl - Leibniz-Zentrum fur Informatik, 2018.

28. Amert, T., Otterness, N., Yang, M., Anderson, J.H. et al.,, "GPU Scheduling on the NVIDIA TX2: Hidden Details Revealed," in 2017 IEEE Real-Time Systems Symposium, RTSS 2017, Paris, France, December 5-8, 2017, 104-115, IEEE Computer Society, 2017.

(c) 2021 Hamid Tabani, Leonidas Kosmidis, Enrico Mezzetti, Jaume Abella, Francisco J. Cazorla. Published by SAE International. This Open Access article is published under the terms of the Creative Commons Attribution License (http://creativecommons.org/licenses/by/4.0/), which permits distribution, and reproduction in any medium, provided that the original author(s) and the source are credited.

Positions and opinions advanced in this work are those of the author(s) and not necessarily those of SAE International. Responsibility for the content of the work lies solely with the author(s).

ISSN 0148-7191 